\title{
Combining School and Family-Centered Interventions to Prevent Child Behavior Problems
}

Elise N. Robeson, B.S., Joni D. Splett, B.A., \& Wendy M. Reinke, Ph.D.

\section{Abstract}

Although school-wide PBS has been shown to reduce problem behaviors among students, approximately $15-20 \%$ of students are still in need of more selective interventions due to disruptive behavior problems. Given that both school (see Reinke \& Herman, 2002) and family factors (Banks, et al., 1993 contribute to behavior problems in children, combining interventions that target both the school environment and family context is expected to further improve outcomes for those students. An ecological model combining PBS and the Family Check-up is proposed to prevent and reduce behavior problems in children.

\section{PBS}

PBS is a three tiered, school-wide continuum service model whereby all students receive universal prevention support marked by consistent rules and consequences, encouragement, and clear expectations across all school settings (Sugai, Sprague, Horner, \& Walker, 2000). Students with at-risk or more severe behaviors may receive selective or indicated interventions to address these concerns. At all three levels, PBS focuses on behavior within environmental contexts by targeting multiple systems within the school including classrooms, non-classroom settings, and individual student and adult staff behavior. Schools across the United States have implemented PBS, and have shown a reduction in problem behaviors among students (Lewis \& Sugai, 1999). Additionally, PBS has been shown to be effective in improving social (Irvin et al, 2004; Taylor-Green et. al., 1997) and academic behavior (McIntosh, Chard, Boland, \& Horner, 2006).

\section{The Family Check-Up}

The Family Check-up (FCU) is a three-tiered family intervention that provides supports along a service continuum, similar to the PBS model (Dishion \& Kavanaugh, 2003; Dishion \& Stormshak, 2007). At the universal level, all families are provided basic services and support via a school Family Resource Center. At the selected and indicated levels, at-risk students are identified and families are offered a three session FCU. The three sessions consist of: (1)Initial intake interview, including questionnaire completion, identification of concerns and goals; (2) Ecological assessment, including gathering information from multiple sources regarding peer relations, family context, and child adjustment; and (3) Feedback and motivation, where results are shared with parents and an intervention menu is presented. The efficacy of the FCU has been proven in multiple studies and results suggest reduced student involvement with deviant peers, lower levels of substance use, and increased parental participation (Stormshack, Dishion, Light, \& Yasui, 2005; Connell, Dishion, Yasui, \& Kavanagh, 2007).

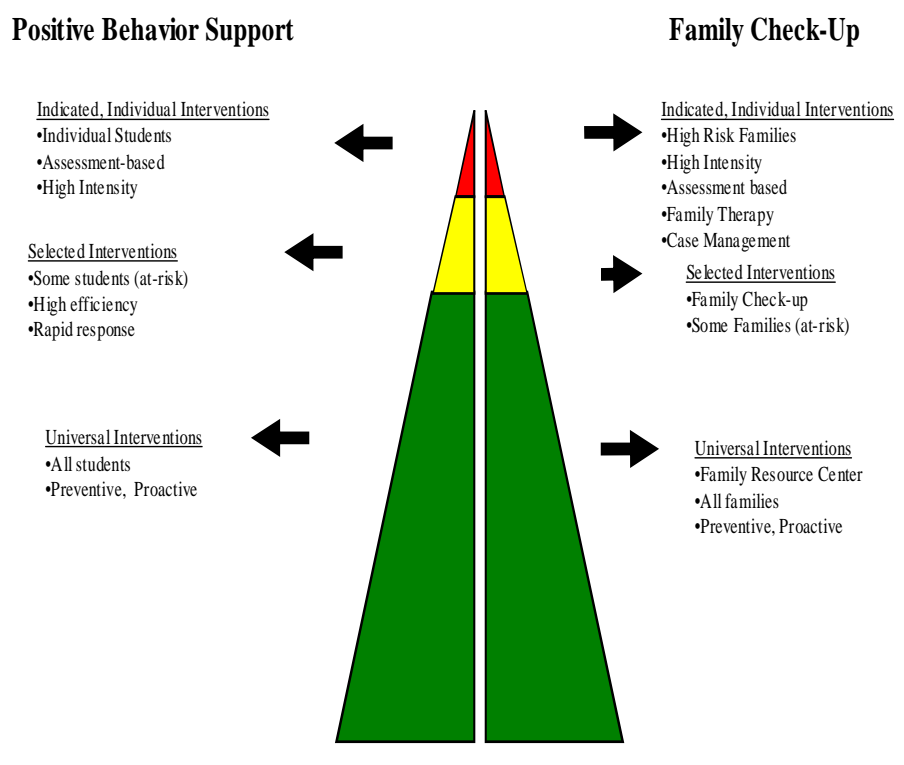

Integration of PBS and FCU

The combination of PBS and FCU has the potential to be a successful program for preventing and reducing problem behaviors. Both interventions operate from an ecological perspective and provide a continuum of supports to all students and families. Within this merged model, family-based services would operate within a Family Resource Center located within the school building (see Dishion \& Kavanaugh, 2003). Following a continuum of support, services offered via the Family Resource Center would include:

$\sim$ Universal: parenting tips and strategies

$\sim$ Selective \& Indicated: family assessments of both strengths and weaknesses within the family unit; menu of services for family support that can be chosen by the family to meet their specific needs

Ongoing PBS data collection (e.g., office discipline referrals) would provide information about students who could be possible candidates for the FCU, as well as provide ongoing monitoring to determine the effectiveness of family-based interventions. Integrating the FCU within PBS would expand PBS beyond the school to target family systems, while increasing the effectiveness of the two interventions and offering unique contribution to the field of school psychology and the overall prevention of disruptive behavior problems in children.

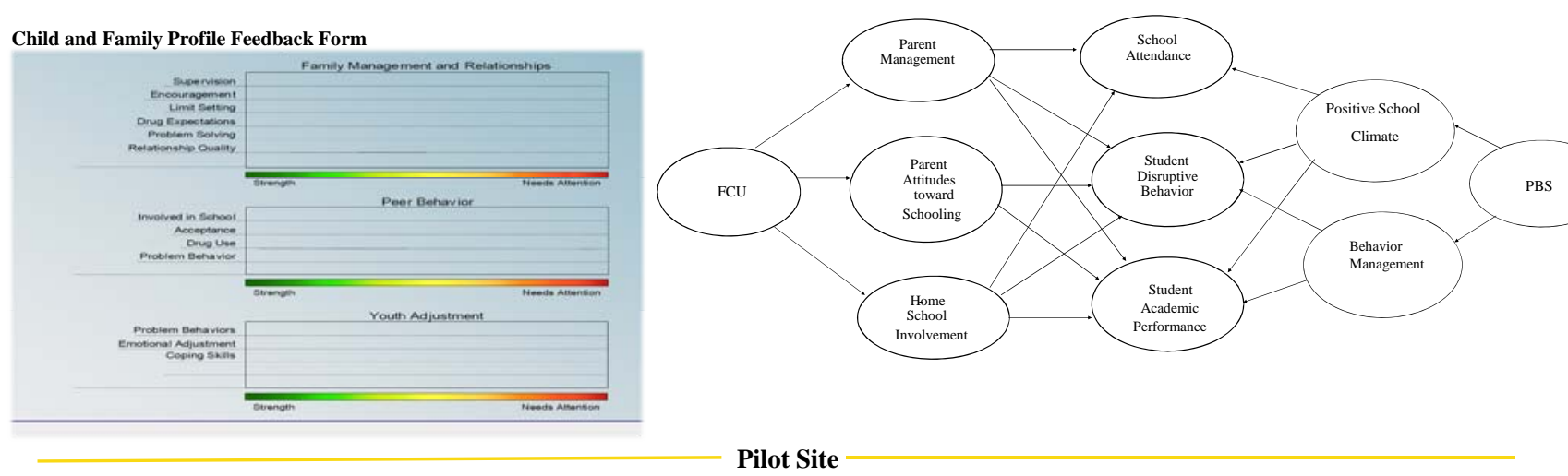

A pilot site will utilize this model within a school-based Family Resource Center (FRC) in a small, predominantly Caucasian, rural Missouri town. The school district has a total of 1400 students and contains one primary school (K-2), one elementary school (3-5), one middle school (6-8), and one high school (9-12). The FRC will be housed in the primary and elementary schools, totaling 600 students. Within the first phase of the project, the FRC will be established and universal information will be provided to all parents. Utilizing PBS data, students will be identified as candidates for the FCU and families will be contacted to discuss student concerns and the FCU intervention. 